\title{
DataMining a Keystroke Dynamics Based Biometrics Database Using Rough Sets
}

\author{
Kenneth Revett, Sérgio Tenreiro de Magalhães and Henrique Santos, Member, IEEE
}

\begin{abstract}
Software based biometrics, utilising keystroke dynamics has been proposed as a cost effective means of enhancing computer access security. Keystroke dynamics has been successfully employed as a means of identifying legitimate/llegitimate login attempts based on the typing style of the login entry. In this paper, we collected keystroke dynamics data in the form of digraphs from a series of users entering a specific login ID. We wished to determine if there were any particular patterns in the typing styles that would indicate whether a login attempt was legitimate or not using rough sets. Our analysis produced a sensitivity of $96 \%$, specificity of $93 \%$ and an overall accuracy of $95 \%$. The results of this study indicate that typing speed and the first few and the last few characters of the login ID were the most important indicators of whether the login attempt was legitimate or not.
\end{abstract}

Index Terms - Artificial Intelligence, Decision Support Systems, Genetic Algorithms

\section{INTRODUCTION}

Keystroke dynamics was first introduced in the early 1980s as a method for identifying the individuality of a given sequence of characters entered through a traditional computer keyboard. Researchers focused on the keystroke pattern, in terms of keyboard duration and keyboard latency $[1,2,3]$. Evidence from preliminary studies indicated that when two individuals entered the same login details, their typing patterns would be sufficiently unique as to provide a characteristic signature that could be used to differentiate one from the another. If one of the signatures could be definitively associated with a proper user, then any differences in typing patterns associated with that particular login ID/password must be the result of a fraudulent attempt to use those details. Thus, the notion of a software based

$K$. Revett is with the University of Westminster, Harrow School of Computer Science, Harrow, London, England HA1 3TP (phone: +442079115000; fax: +442079115608; e-mail: revettk@westminster.ac.uk).

S. Tenreiro de Magalhães is with the Universidae de Minho, Department of Information SystemsCampus de Azurem 4800-058 Guimaraes, Portugal (email: psmagalhaes@dsi.uminho.pt).

H. Santos is with the Universidae de Minho, Department of Information Systems Campus de Azurem 4800-058 Guimaraes, Portugal (email:. (e-mail: hsantos@dsi.uminho.pt). biometric security enhancement system was born. Indeed, there are commercial systems such as BioPassword that have made use of this basic premise.

Deterministic algorithms have been applied to keystroke dynamics since the late 70's. In 1980 Gaines [1] presented a report of his work to study the typing patterns of seven professional typists. The small number of volunteers and the fact that the algorithm is deduced from their data and not tested in other people later, results on a lower confidence on the FAR and FRR values presented. But the method used to establish a pattern was a breakthrough: a study of the time spent to type the same two letters (digraph), when together in the text. Since then, many algorithms based on Algebra and on Probability and Statistics have been presented. Joyce and Gupta presented in 1990 [2] an algorithm to calculate a value that represents the distance between acquired keystroke latency times and correspondent times previously stored. In 1997 Monrose and Rubin use the Euclidean Distance and probabilistic calculations based on the assumption that the latency times for one-digraph exhibits a Normal Distribution [4]. Later, in 2000, they also present an algorithm for identification, based on the similarity models of Bayes [5], and in 2001 they present an algorithm that uses polynomials and vector spaces to generate complex passwords from a simple one, using the keystroke pattern. In 2005 Magalhães and Santos [3] presented an improvement of the Joyce and Gupta's algorithm, while Revett and Khan [6] presented evidence of the existence of a set of procedures (typing rhythms, length of the password, etc.) that can enhance the precision of these algorithms. In this study, we employ a rough sets based classifier in order to determine which attributes in the input signature are important to the identification of a legitimate owner of a login ID sequence.

The rough set theory, proposed by Pawlak [7,8], is an attempt to propose a formal framework for the automated transformation of data into knowledge. It is based on the idea that any inexact concept (for example, a class label) can be approximated from below and from above using an indiscernibility relationship (generated by information about objects). Pawlak [7] points out that one of the most important and fundamental notions to the rough sets philosophy is the need to discover redundancy and dependencies between features. Since then this philosophy has been used successfully in several tasks as, for example, construction of rule based classification schemes, identification and evaluation of data dependencies, information-preserving data reduction $[8,9,10]$. 
Table 1. This table presents a sample of 5 legitimate users (' 1 ' in the Legit? column) and 5 illegitimate users (with a ' 0 ' in the Legit? column). All other values in the table are the digraph times in $\mathrm{mS}$. The ellipsis indicate that values were not included because of space constraints only

\begin{tabular}{||r||r||c||c||c||c||}
\hline T1 & T2 & $\ldots$ & T12 & T13 & Legit? \\
\hline \hline 281 & 344 & $\ldots$ & 282 & 281 & 1 \\
\hline \hline 343 & 266 & $\ldots$ & 282 & 250 & 1 \\
\hline \hline 375 & 359 & $\ldots$ & 344 & 359 & 1 \\
\hline \hline 250 & 328 & $\ldots$ & 297 & 235 & 1 \\
\hline \hline 391 & 250 & $\ldots$ & 265 & 438 & 1 \\
\hline \hline 390 & 344 & $\ldots$ & 453 & 235 & 0 \\
\hline \hline 546 & 625 & $\ldots$ & 500 & 219 & 0 \\
\hline \hline 344 & 359 & $\ldots$ & 438 & 234 & 0 \\
\hline \hline 531 & 501 & $\ldots$ & 328 & 297 & 0 \\
\hline \hline 390 & 344 & $\ldots$ & 532 & 265 & 0 \\
\hline
\end{tabular}

In this work, we wished to determine if any of the attributes in the decision table (see Table 1 below) were superfluous and consequently could be removed without affecting the accuracy of the classification task: namely to determine if a user was the legitimate owner of a login ID or not. In this way, we would be able to reduce the amount of information that was collected when a user logged into our system - focusing only on what was essential. This would reduce the computational load on any system designed to detect intruder entry. In the next section of this paper, we describe the data that we analysed and our rough sets based analysis, followed by some of the key results from our rough sets based analysis, and lastly a brief discussion of our results.

\section{METHODS}

In this study, we asked users (approximately 100) to enter a passphrase that consisted of a string of 14 characters through an Internet based portal. One user was selected as the owner of this passphrase and was asked to enter the passphrase on numerous occasions (approximately 100). The entries were collected over a one-month time period to ensure that we acquired a robust sampling of the variations of the input style for passphrase entry. We custom designed software (written in Java) that would capture the digraph time - the time when the user depressed each key in the passphrase, resulting in a total of 13 digraphs for the passphrase. The digraphs entries (192 were employed in this study, with 96/96 legitimate/illegitimate entries) formed a decision table with thirteen attributes and a binary decision class (' 1 ' for legitimate and ' 0 ' for illegitimate). We then discretised the attributes (except for the decision attribute) using an entropy based algorithm prior to applying the rough set algorithm written in $\mathrm{C}++$ ). We split the data table into a 70/30 split (134/58) training/testing and repeated this procedure 20 times, with replacement for all subsequent rough sets based analysis, and pooled the results. We applied a dynamic reduct algorithm to find the reducts from the decision table as in [6]. We generated rules using the order based genetic algorithm of [11] from the reducts. We filtered the rules such that we removed all rules with a support less than 5 instances. This was accomplished without a significant reduction in the classification accuracy (see Table 4 for details). In the next section, we present the overall methodology employed and the key results obtained from this study.

\section{RESULTS}

As a first pre-processing step, we discretised the data using an entropy preserving algorithm. After discretisation, the decision table was split into a 70/30 partitioning - repeated 20 times for each of the subsequent analysis steps. Next we used a dynamic reduct algorithm to find the reducts. This stage removes the redundancy in the data while preserving the disceriibility relation between the attributes and their respective decision class. Lastly, we generated the decision rules using an order based genetic algorithm. Without any filtering, a total of 1747 rules were generated from the decision table. A filter was applied, based on support where any object that had a support less than 5 was removed from the rule table. This filtering was applied to both the right hand (RHS) and left hand (LHS) elements of the rules. This reduced the total number of rules down to 657, a large but manageable number. The reduction in rules via filtering did not appreciably reduce the classification accuracy (see table 4 for details). In Table 2 a single confusion matrix is presented (selected randomly from the pool of 20 produced) which indicates a high accuracy level (95\%) when using the filtered rule set (the unfiltered accuracy was 98\%). The final result obtained - and the most important result from this study was the rule set. In Table 3 we present a sample of rules from three different partitions of the data: i) rules selected randomly from the legitimate login attempts, ii) rules from illegitimate login attempts, and iii) non-deterministic rules which are a combination of legitimate and illegitimate login attempts. Lastly, in Table 4 we present data on the integrity/accuracy of our rules set.

Table 2. A sample confusion matrix for a randomly selected application of the rule set generated using rough sets. The top entry in the $3^{\text {rd }}$ column is the sensitivity, the value below that is the specificity. The entry at the bottom of column two is the positive predictive value (PPV), the last entry in column three is the predictive negative value (PNV) and the lower right hand corner is the overall classification accuracy.

\begin{tabular}{|l|l|l|ll|}
\hline Outcomes & 0 & 1 & & \\
\hline 0 & 29 & 1 & $0.96 \quad$ (SE) \\
\hline 1 & 2 & 26 & $0.93 \quad$ (SP) \\
\hline & 0.94 & 0.96 & $\mathbf{0 . 9 5}$ accuracy) \\
& (PPV) & (PNV) & & \\
\hline
\end{tabular}


Table 3. A set of 8 rules that were generated using filtering on support $>=5$ entries. Note that there is a mixture of deterministic (with a single decision ' 1 ' or ' 0 ') and nondeterministic rules with two decisions: ' 1 ' and ' 0 '. The '*' refers to either 0 if it appears on the left of a tuple, or the end result if it appears on the right end of a tuple. All rules are generated in conjunctive normal form

\begin{tabular}{|c|c|}
\hline Rule & Decision \\
\hline $\begin{array}{l}\text { Time } 1([*, 407)) \text { AND Time } 2([*, 383)) \\
\text { AND Time } 4([*, 305)) \text { AND Time } \\
10([*, 391))=>\end{array}$ & 1 \\
\hline $\begin{array}{l}\text { Time } 1([*, 407)) \text { AND Time } 9([*, 243)) \\
\text { AND Time } 10([*, 391))=>\end{array}$ & 1 \\
\hline $\begin{array}{l}\text { Time } 2([*, 383)) \text { AND Time } 3([*, 508)) \\
\text { AND Time } 10\left(\left[\left[^{*}, 391\right)\right) \text { AND Time }\right. \\
11([*, 524))=>\end{array}$ & 0 and 1 \\
\hline $\begin{array}{l}\text { Time } 3([*, 508)) \text { AND Time } 5\left(\left[\left[^{*}, 321\right)\right)\right. \\
\text { AND Time } 10\left(\left[\left[^{*}, 391\right)\right) \text { AND Time }\right. \\
13([*, 329))=>\end{array}$ & 0 and 1 \\
\hline $\begin{array}{l}\text { Time } 5\left(\left[{ }^{*}, 321\right)\right) \text { AND Time } 10\left(\left[^{*},\right.\right. \\
\text { 391)) AND Time } 11([*, 524)) \text { AND } \\
\text { Time } 13([*, 329))=>\end{array}$ & 0 and 1 \\
\hline $\begin{array}{l}\text { Time } 3([*, 508)) \text { AND Time } 8([*, 305)) \\
\text { AND Time } 10([*, 391)) \text { AND Time } \\
13([*, 329))=>\end{array}$ & 0 and 1 \\
\hline $\begin{array}{l}\text { Time } 1([586, *)) \text { AND Time } 4([336, \\
774)) \text { AND Time } 5([*, 321)) \text { AND Time } \\
10([438,1367))=>\end{array}$ & 0 \\
\hline $\begin{array}{l}\text { Time } 1([407,571)) \text { AND Time } 4([336, \\
774)) \text { AND Time } 5([*, 321)) \text { AND Time } \\
10([438,1367))=>\end{array}$ & 0 \\
\hline
\end{tabular}

In addition to the actual rules that are generated from the application of the reducts to the decision table, there are a number of criteria that are used to judge the applicability/accuracy of the rules: support and accuracy. The support refers to the number of instances in the table in which a given antecedent maps to the same decision value. The accuracy is a measure of how well the decision classes are generated given the evidence (the values of the antecedents). These measures of classification accuracy, support and accuracy are depicted in Table 4 below.

\section{DISCUSSION}

In this pilot study, we used rough sets to mine a small database of keystroke based biometric data - using only digraph times. We generated a decision table by including the correct decision class (legitimate or illegitimate owner) and were able to predict with a high degree of accuracy whether the attempt was legitimate or not based on the decision rules that we generated from rough sets $(95 \%$ or more classification accuracy). The most interesting result from this study indicate
Table 4. A listing of the classification accuracy measurements (support and accuracy) for the rules that are listed in Table 3 above (listed in the same order as the rules). The 'Accuracy' column indicates the accuracy for the specified decision class(es). The numeric values in the 'Support' column heading indicate the number of instances for each decision rule

\begin{tabular}{|cc|cc|}
\hline \multicolumn{2}{|c|}{ Support } & \multicolumn{2}{c|}{ Accuracy } \\
\hline LHS & RHS & Decision: 1 & 0 \\
\hline 55 & 55 & $100 \%$ & $0 \%$ \\
\hline 59 & 59 & $100 \%$ & $0 \%$ \\
\hline 49 & 48,1 & $97.9 \%$ & $2.1 \%$ \\
\hline 39 & 38,1 & $97.4 \%$ & $2.6 \%$ \\
\hline 41 & 40,1 & $97.6 \%$ & $2.4 \%$ \\
\hline 43 & 42,1 & $97.7 \%$ & $2.3 \%$ \\
\hline
\end{tabular}

that the digraph times (see Table 4 for details of the rules) was most critical for determining whether a user was legitimate. As can be seen in Table 3, the decision class labelled ' 1 ' - the legitimate owner - took the least amount of time in entering the characters of their login ID compared with that of an illegitimate owner. In addition, the legitimate owner of the login ID, the first few and last digraphs were sufficient to make a correct classification. This implies that instead of using all of the digraphs in a signature for verification, we may only require a subset of them - depending on the particular login ID characteristics of the owner. This reduction in the number of attributes that must be stored and searched through reduces the computational load of the verification system. The use of rules generated from rough sets based classifiers can be enhanced by the addition of more attributes into the decision table. With these encouraging results, we are expanding our analysis using much larger datasets, both in terms of the number of objects, but also by the inclusion of additional attributes. We hope to discover what attributes are critical for particular login IDs in order to tailor the system so that it can emphasise those keystroke dynamic features that are indicative of the legitimate owner.

\section{REFERENCES}

[1] Gaines, R. et al. Authentication by keystroke timing: Some preliminary results. Rand Report R-256-NSF. (1980) Rand Corp

[2] Joyce, R. and Gupta, G.. Identity authorization based on keystroke latencies. Communications of the ACM. Vol. 33(2), (1990) pp 168-176.

[3] Magalhães, S. T. and Santos, H. D., 2005, An improved statistical keystroke dynamics algorithm, Proceedings of the IADIS MCCSIS 2005.

[4] Monrose, F. and Rubin, A. D., 1997. Authentication via Keystroke Dynamics. Proceedings of the Fourth ACM Conference on Computer and Communication Security. Zurich, Switzerland.

[5] Monrose, F. and Rubin, A. D., 2000. Keystroke Dynamics as a Biometric for Authentication. Future Generation Computing Systems (FGCS) Journal: Security on the Web.

[6] Revett, K. and Khan, A., 2005, Enhancing login security using keystroke hardening and keyboard gridding, Proceedings of the IADIS MCCSIS 2005. 
[7] Ørn, A.. "Discernibility and Rough Sets in Medicine" Tools and Applications. Department of Computer and Information Science. Trondheim, Norway, Norwegian University of Science and Technology: 239, 1999.

[8] Pawlak, Z. Rough Sets, International Journal of Computer and Information Sciences, 11, (1982) pp. 341- 356.

[9] Pawlak, Z.: Rough sets - Theoretical aspects of reasoning about data. Kluwer (1991).

[10] Slezak, D.: Approximate Entropy Reducts. Fundamenta Informaticae (2002).

[11] Wroblewski, J.: Theoretical Foundations of Order-Based Genetic Algorithms. Fundamenta Informaticae 28(3-4) (1996) pp. 423-430. 